\title{
Expression of semaphorin 6D and its receptor plexin-A1 in gastric cancer and their association with tumor angiogenesis
}

\author{
YANJIE LU ${ }^{1,2^{*}}$, QIAN XU ${ }^{2,3^{*}}$, LEI CHEN ${ }^{4}$, YANZHEN ZUO ${ }^{2,5}$, SHAOCHEN LIU ${ }^{1,2}$, \\ YATAO HU ${ }^{1,2}$, XIAORU LI ${ }^{6}$, YUHONG LI ${ }^{1,2}$ and XIANGYANG ZHAO ${ }^{2,4}$ \\ ${ }^{1}$ Department of Pathology; ${ }^{2}$ Cancer Research Laboratory; ${ }^{3}$ Institute of Basic Medical Sciences, Chengde Medical College; \\ ${ }^{4}$ Department of General Surgery, The 266th Hospital of Chinese People's Liberation Army; ${ }^{5}$ Department of Pharmacology, \\ Chengde Medical College; ${ }^{6}$ Department of Gynecology and Obstetrics, The Central Hospital of Chengde City, \\ Chengde, Hebei 067000, P.R. China
}

Received June 23, 2015; Accepted September 2, 2016

DOI: $10.3892 / \mathrm{ol} .2016 .5208$

\begin{abstract}
The semaphorin and plexin family of ligands and receptor proteins provides important axon growth and guidance cues required for development. In recent years, studies have expanded their role in the regulation of cardiac morphogenesis and tumorigenesis. However, the mechanism responsible for their role in regulating cancer development and progression has not been clarified. In the present study, semaphorin 6D (Sema6D) and its receptor plexin-A1 were identified to be expressed at high levels in vascular epithelial cells within gastric cancer, and were positively correlated with vascular endothelial growth factor receptor 2 (VEGFR2). These findings verify our hypothesis that Sema6D and plexin-A1 may be closely associated with tumor angiogenesis. Combined with experimental observations in the MGC803 gastric cancer cell line, it was observed that knocking down plexin-A1 signaling led to a decreased expression of VEGFR 2 at the messenger RNA and protein levels. Sema6D recognized and activated plexin-A1, which subsequently activated its downstream target, VEGFR2. The activation of VEGFR2 functioned as a positive regulator of tumor angiogenesis. Our data provided an understanding of the complex signaling cascades involved in the angiogenesis-related pathway in tumor cells. In light of our observations, pharmacological interventions targeting
\end{abstract}

Correspondence to: Professor Yuhong Li, Department of Pathology, Chengde Medical College, Shangerdaohezi Avenue, Chengde, Hebei 067000, P.R. China

E-mail: youngcheer2003@foxmail.com

Professor Xiangyang Zhao, Department of General Surgery, The 266th Hospital of Chinese PLA, Puning Avenue, Chengde, Hebei 067000, P.R. China

E-mail: luyanjiehappy@163.com

*Contributed equally

Key words: semaphorin 6D, plexin-A1, VEGFR2, gastric cancer, angiogenesis
Sema6D/plexin-A1/VEGFR2 signaling may potentially be used as a target for the development of novel anti-angiogenic drugs in gastric cancer.

\section{Introduction}

The semaphorin family is a large super-protein family containing secreted, transmembrane and glycosylphosphatidylinositol-linked proteins, and it has been divided into eight hypotypes based on their structures and amino acid sequence similarity: Invertebrate semaphorins consist of classes 1 and 2, while classes 3-7 are vertebrate semaphorins (with the exception of class 5C semaphorins, which are encoded by viral genomes) (1). The semaphorin family was initially identified to be involved in mediating axonal guidance in the developing nervous system (2). Certain members of the semaphorin family have been also shown to exert diverse and important functions in other physiological processes, including heart morphogenesis $(3,4)$, vascular growth (5), immune cell regulation (6) and tumor progression $(7,8)$. Important regulatory functions of certain members of the semaphorin family within tumor angiogenesis have been reported. For example, semaphorin 3A (Sema3A) inhibits angiogenesis through competition for vascular endothelial growth factor (VEGF) (9), and Sema3B has also been indicated as a putative tumor suppressor that inhibits tumor growth and angiogenesis (10). By contrast, Sema3C presumably may promote angiogenesis by stimulating integrin phosphorylation and VEGF120 secretion (11), and Sema4D has been demonstrated to serve a role in tumor-induced angiogenesis (12).

Sema6D, mapped on chromosome 2, is the best characterized factor of the class 6 semaphorins, which are single-pass membrane-bound semaphorins (13). It was reported that Sema6D regulates the late-phase activity of T cells during the primary immune response (14), and it functions as a promoter of tissue remodeling (15) and tumorigenesis (16). Additionally, Sema6D also functions as a ligand for plexin-A1 during $\mathrm{T}$ cell-dendritic cell interactions (6). Plexin-A1 serves as a main receptor for Sema6D and contributes to cardiac morphogenesis (17). Notably, plexin-A1 forms complexes with VEGF receptor-2 (VEGFR-2), which undergoes phosphorylation 
Table I. Primers used in the present study.

\begin{tabular}{ll}
\hline Transcript & \multicolumn{1}{c}{ Primer sequence (5'-3') } \\
\hline Sema6D & $\begin{array}{l}\text { TGAGGAGGAAGGTAGCTCAGTG (Sense) } \\
\text { CCATCAGCAGCAGTATGTAGGC (Antisense) }\end{array}$ \\
Plexin-A1 & $\begin{array}{l}\text { TGGACGACCTGTTTAGACCA (Sense) } \\
\text { TGATCACGTTCACCCAGAAGC (Antisense) }\end{array}$ \\
Plexin-A2 & $\begin{array}{l}\text { CATCYCGTACTGGACCCCAC (Sense) } \\
\text { TTTACAACGGCTACAGCGTG (Antisense) }\end{array}$ \\
Plexin-A4 & TCTCAGTACAACGTGCTG (Sense) \\
VAGCACTGGATCTGATTGC (Antisense) & CTACCAGTACGGCACCACTCAA (Sense) \\
VE-actin & $\begin{array}{l}\text { TCTTCCTCCAACTGCCAATACC (Antisense) } \\
\text { TGACGTGGACATCCGCAAAG (Sense) }\end{array}$ \\
CTGGAAGGTGGACAGCGAGG (Antisense) & $\begin{array}{l}\text { TGAAGGTCGGAGTCAACGGAT (Sense) } \\
\text { CTGGAAGATGGTGATGGGATT (Antisense) }\end{array}$
\end{tabular}

Sema, semaphorin; VEGFR, vascular endothelial growth factor receptor; GAPDH, glyceraldehyde 3-phosphate dehydrogenase.

upon stimulation by Sema6D (15), and VEGFR is responsible for the transduction of pro-angiogenic signals (18). The association between plexin-A1 expression and gastric carcinoma angiogenesis has been previously explored by the present authors (19).

In the current study, Sema6D was observed to be highly expressed in tumor tissue compared with normal gastric mucosa and was determined to be responsible for tumor promotion. Its putative receptors were detected, and it was speculated that plexin-A1 may be the main receptor for Sema6D. Next, the levels of Sema6D and plexin-A1 were detected, and it was noticed that they were highly expressed in tumor vascular endothelial cells. Furthermore, both were positively correlated with VEGFR2. These observations indicate that they may function as a modifier in the formation of tumor angiogenesis. This is consistent with a previous report showing that Sema6D activates VEGFR2 through plexin-A1-mediated signal transduction and controls cardiac morphogenesis (20).

\section{Materials and methods}

Human tissue specimens. Gastric tissues were obtained from The 266th General Hospital of People's Liberation Amy (Chengde, China) with the institutional approval and informed consent of the patients. The procedures to obtain human gastric tissues were in accordance with the Ethical Principles for Medical Research Involving Human Subjects, as formulated in the World Medical Association Declaration of Helsinki (revised in 2008). During surgical resection, gastric tumor tissues and normal gastric tissues (located $\sim 5 \mathrm{~cm}$ away from the macroscopic margin of the resected tumors) were obtained from 10 patients who were diagnosed as gastric carcinomas by pathologists. The patient ages ranged between 37 and 82 years, with a median age of 58 years. There were six male and four female patients. None of the patients had received any chemotherapy or radiotherapy prior to biopsy or surgery.
Reagents. RPMI-1640 medium, fetal bovine serum (FBS), $0.25 \%$ trypsin and $0.02 \%$ ethylenediaminetetraacetic acid (EDTA) were purchased from Gibco (Thermo Fisher Scientific, Inc., Waltham, MA, USA). SuperScript III FirstStrand Synthesis System kit and GoTaq ${ }^{\circledR}$ qPCR Master Mix were purchased from Promega Corporation (Madison, WI, USA). Anti-VEGFR2 mouse monoclonal antibody (ab9530), anti-plexin-A1 rabbit monoclonal antibody (ab32960) and anti- $\beta$-actin mouse monoclonal antibody (ab8226) were purchased from Abcam (Cambridge, MA, USA), while anti-Sema6D goat monoclonal antibody (sc-67965) was purchased from Santa Cruz Biotechnology, Inc. (Dallas, TX, USA). Peroxidase-conjugated AffiniPure goat anti-mouse immunoglobulin $\mathrm{G}(\mathrm{IgG})$, peroxidase-conjugated AffiniPure goat anti-rabbit IgG, peroxidase-conjugated AffiniPure donkey anti-goat IgG, Alexa Fluor ${ }^{\circledR}$ 488-conjugated AffiniPure goat anti-mouse IgG, Alexa Fluor ${ }^{\circledR}$ 594-conjugated AffiniPure donkey anti-goat IgG and Alexa Fluor ${ }^{\circledR}$ 594-conjugated AffiniPure goat anti-rabbit IgG were purchased from Santa Cruz Biotechnology, Inc.

Cell culture. Human gastric cancer cell lines (MGC803, HGC27 and MNK45) and human normal gastric mucosa cell line (GES-1) were provided by the Academy of Military Medical Sciences (Beijing, China). The cell lines were cultured in an incubator with an atmosphere of $5 \% \mathrm{CO}_{2}$ at $37^{\circ} \mathrm{C}$ in RPMI-1640 medium supplemented with 10\% FBS. The cells were then subcultured with $0.25 \%$ trypsin and $0.02 \%$ EDTA when the cell growth reached $80-90 \%$. The experiments were carried out when the cells reached logarithmic growth phase.

Reverse transcription-quantitative polymerase chain reaction (RT-qPCR) analysis. Total RNA was isolated using the RNAgents ${ }^{\circledR}$ Total RNA Isolation System (Promega Corporation) with DNase I (Invitrogen; Thermo Fisher Scientific, Inc.) treatment. RNA $(2 \mu \mathrm{g})$, oligo(dT)20 primers and the SuperScript III First-Strand Synthesis System kit were used to synthesize complementary DNA. qPCR was performed using the $\mathrm{SYBR}^{\circledR}$ Green I dye provided in the GoTaq ${ }^{\circledR}$ qPCR Master Mix according to the manufacturer's protocol. PCR was performed under the following conditions: Denaturation at $95^{\circ} \mathrm{C}$ for $30 \mathrm{sec}$, followed by 40 cycles at $95^{\circ} \mathrm{C}$ for $3 \mathrm{sec}$, $60^{\circ} \mathrm{C}$ for $30 \mathrm{sec}$ and $72^{\circ} \mathrm{C}$ for $45 \mathrm{sec}$. The results were analyzed using the comparative quantitative cycle $(\mathrm{Cq})$ method (21), with glyceraldehyde 3-phosphate dehydrogenase (GAPDH) as an internal control. The results were normalized to the GAPDH levels using the formula $\Delta \mathrm{Cq}=\mathrm{Cq}$ of the target gene $-\mathrm{Cq}$ of GAPDH. The messenger RNA (mRNA) level of the control group was used as the baseline, and $\Delta \Delta \mathrm{Cq}$ was calculated using the formula $\Delta \Delta \mathrm{Cq}=\Delta \mathrm{Cq}$ of the target gene $-\Delta \mathrm{Cq}$ of the baseline. The fold-change in mRNA level was calculated as $2^{-\Delta \Delta \mathrm{Cq}}$.

For RT-PCR, the PCR products were resolved in $2 \%$ agarose gels and visualized by staining with ethidium bromide. To semiquantify the PCR products, the bands representing the amplified products were analyzed by Quantity One ${ }^{\circledR} 1-\mathrm{D}$ analysis software (Bio-Rad Laboratories, Inc., Hercules, CA, USA). The relative level of the target mRNA expression was defined as the ratio of the absorbance of the target band to that of the $\beta$-actin band. The primers used in the present study 
A

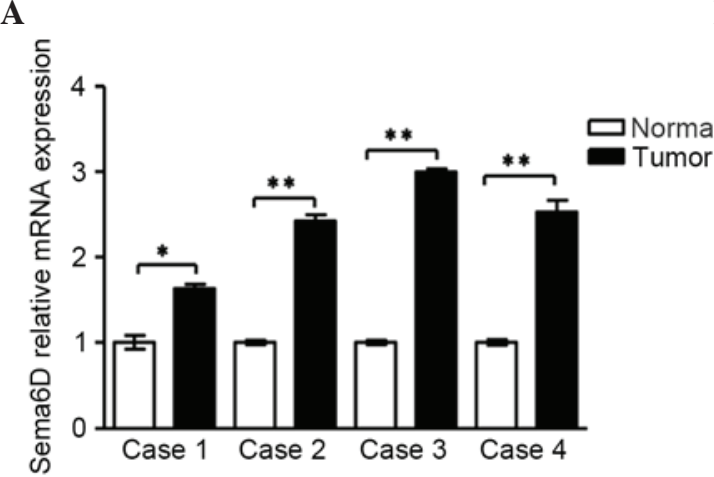

B

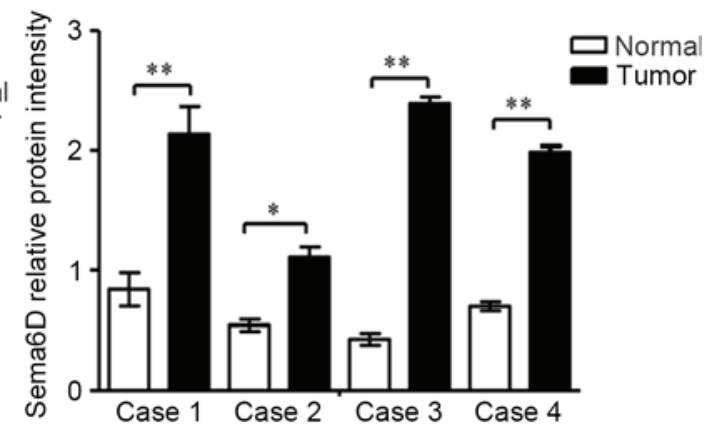

C

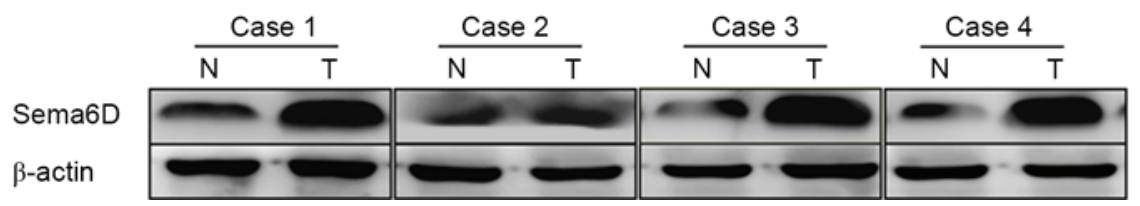

Figure 1. Sema6D was highly expressed in four cases of gastric cancer tissue. (A) Reverse transcription-quantitative polymerase chain reaction analyses of the mRNA expression of Sema6D in four cases of gastric cancer. Sema6D mRNA levels increased remarkably by 1.6, 2.4, 2.9 and 2.6-fold in tumor cases 1, 2, 3 and 4, respectively, when compared with normal gastric mucosa. (B) The relative protein intensities of Sema6D with respect to the loading control, $\beta$-actin, are shown. (C) The protein expression levels of Sema6D in the four specimens were enhanced. Data are represented as the mean \pm standard deviation ( $\mathrm{n}=3$ for each group, $\left.{ }^{*} \mathrm{P}<0.05,{ }^{* *} \mathrm{P}<0.01\right)$. Sema, semaphorin; mRNA, messenger RNA; N, normal; T, tumor.

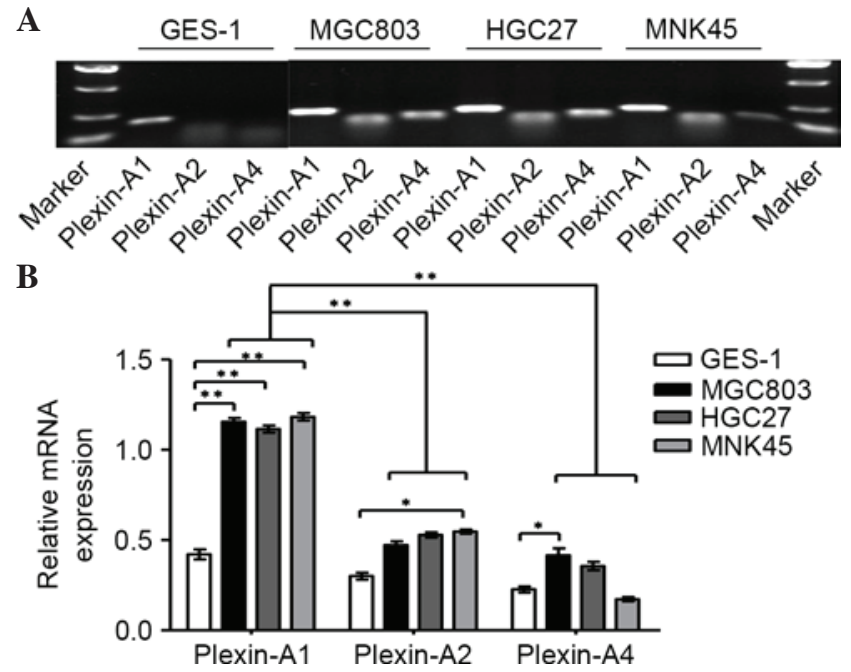

Figure 2. Expression of the receptor isoforms for semaphorin 6D in gastric cancer cell lines. (A) Reverse transcription-polymerase chain reaction was performed to evaluate the expression pattern of plexin-A isoforms in three gastric cancer cell lines and GES-1 cells. Putative plexin-A isoform bands are shown according to the marker. The sizes of the plexin-A isoforms are as follows: Plexin-A1 (193 bp), plexin-A2 (167 bp) and plexin-A4 (179 bp). (B) The mRNA relative intensities of plexin-As in comparison with the loading control $\beta$-actin are shown. Plexin-A1 was obviously increased in all three gastric cancer cell lines (MGC803, HGC27 and MNK45) compared with GES-1 cells. Data are represented as the mean \pm standard deviation $(n=3$ for each group, $\left.{ }^{*} \mathrm{P}<0.05,{ }^{* *} \mathrm{P}<0.01\right)$. mRNA, messenger RNA.

were synthesized by Shenzhen Huada Gene Technology Co., Ltd. (Shenzhen, China), and their sequences are presented in Table I.

Western blot analysis. Total proteins were extracted from each group with radioimmunoprecipitation assay buffer (Thermo Fisher Scientific, Inc.) and quantified using the Pierce BCA Protein Assay kit (Thermo Fisher Scientific,
Inc.). The proteins were separated by $10 \%$ sodium dodecyl sulfate-polyacrylamide gel electrophoresis and transferred to polyvinylidene difluoride membranes. Upon blocking with $5 \%$ bovine serum albumin (Beijing Solarbio Science \& Technology Co., Ltd., Beijing, China), the blots were probed with the appropriate primary antibodies overnight at $4^{\circ} \mathrm{C}$. The antibodies used were anti-Sema6D antibody (1:500 dilution), anti-plexin-A1 antibody (1:1,000 dilution), anti-VEGFR2 antibody (1:500 dilution) and anti- $\beta$-actin antibody (1:1,000 dilution). The membranes were washed three times for $10 \mathrm{~min}$ in Tris-buffered saline containing Tween 20, and incubated with horseradish peroxidase-conjugated secondary antibodies [goat anti-mouse (sc-2039; Santa Cruz Biotechnology, Inc.), goat anti-rabbit (sc-2040; Santa Cruz Biotechnology, Inc.) or donkey anti-goat (sc-2024; Santa Cruz Biotechnology, Inc.), correspondingly] at $1: 1,000$ dilution for $2 \mathrm{~h}$ at $37^{\circ} \mathrm{C}$. Immunoreactive bands were detected using Pierce ECL Western Blotting Substrate (Thermo Fisher Scientific, Inc.) and imaged using the ImageQuant LAS 4000 system (GE Healthcare Life Sciences, Chalfont, UK).

Immunohistochemistry and fluorescence microscopy. Immunohistochemical analysis was performed using standard techniques. Briefly, paraffin-embedded tissues were cut into 4- $\mu$ m-thick sections, deparaffinized and antigen-recovered in citrate buffer. The sections were blocked for endogenous avidin, peroxidase and biotin, and then incubated with anti-plexin-A1 antibody (ab32960; Abcam) or anti-Sema6D antibody (sc-67965; Santa Cruz Biotechnology, Inc.) overnight at $4^{\circ} \mathrm{C}$. Upon washing three times with phosphate-buffered saline (PBS), the staining was developed using the Universal LSAB $^{\text {Tм }}$ kit/HRP, Rabbit/Mouse/Goat (Dako, Glostrup, Denmark) according to the manufacturer's protocol.

For fluorescence immunohistochemical staining and microscopy, the sections were fixed in $4 \%$ paraformaldehyde for $30 \mathrm{~min}$ and then permeabilized in $0.2 \%$ Triton $\mathrm{X}-100$ in 
A

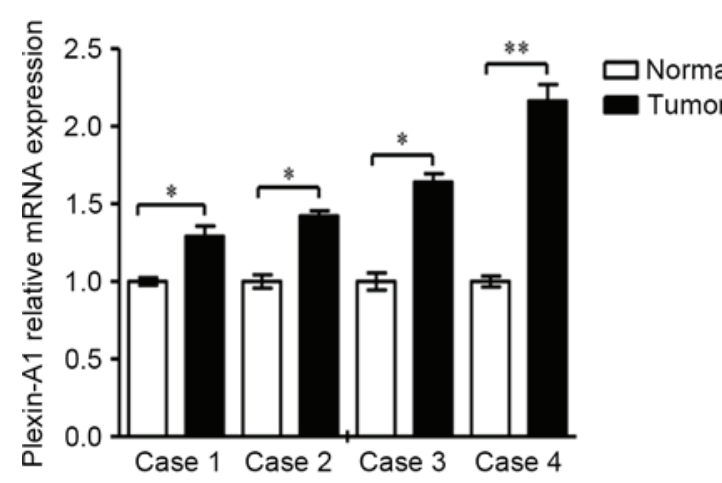

B

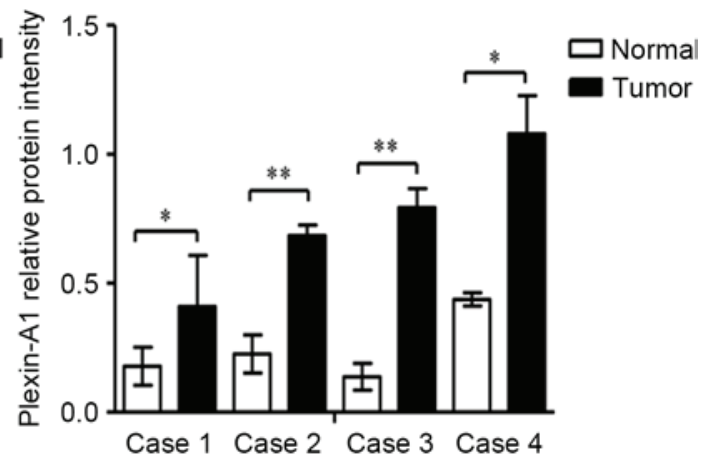

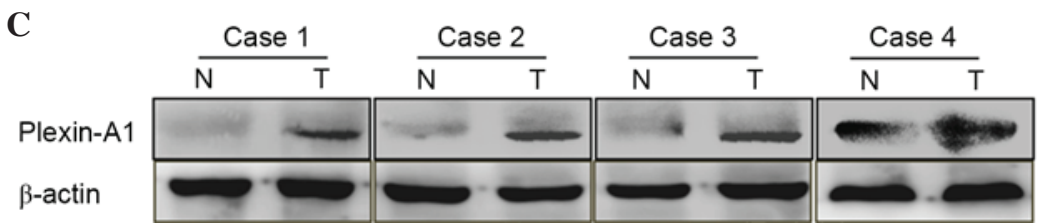

Figure 3. Plexin-A1 was highly expressed in four cases of gastric cancer tissue. (A) The mRNA expression of plexin-A1 was obviously increased in cancer tissue compared with normal gastric mucosa. Plexin-A1 mRNA levels increased remarkably by 1.3, 1.4, 1.6 and 2.2-fold in tumors 1, 2, 3 and 4, respectively, compared with normal gastric tissue. (B) The histogram indicates the protein levels, which were normalized to the levels of $\beta$-actin protein. (C) The protein expression levels of plexin-A1 in all four cancer specimens were remarkably enhanced. Data are represented as the mean \pm standard deviation $(\mathrm{n}=3$ for each group, $\left.{ }^{*} \mathrm{P}<0.05,{ }^{* *} \mathrm{P}<0.01\right)$. mRNA, messenger RNA; N, normal; $\mathrm{T}$, tumor.

PBS for 10 min. The primary antibodies [mouse anti-VEGFR2 antibody (ab9530; Abcam; 1:100 dilution), rabbit anti-plexin-A1 antibody (ab32960; Abcam; 1:200 dilution) and goat anti-Sema6D antibody (sc-67965; Santa Cruz Biotechnology, Inc.; 1:100 dilution)] were incubated overnight at $4^{\circ} \mathrm{C}$. Then, the appropriate Alexa-Fluor ${ }^{\circledR}$-conjugated secondary antibodies [Alexa Fluor ${ }^{\circledR}$ 488-conjugated AffiniPure goat anti-mouse IgG (sc-395764; Santa Cruz Biotechnology, Inc.), Alexa Fluor ${ }^{\circledR}$ 594-conjugated AffiniPure donkey anti-goat IgG (sc-362275; Santa Cruz Biotechnology, Inc.) and Alexa Fluor ${ }^{\circledR}$ 594-conjugated AffiniPure goat anti-rabbit IgG (sc-362282; Santa Cruz Biotechnology, Inc.)] were used at $37^{\circ} \mathrm{C}$ for $2 \mathrm{~h}$ at 1:200 dilution. The nuclei were stained using 4',6-diamidino-2-phenylindole (H-1200; Vector Laboratories, Inc., Burlingame, CA, USA). Fluorescence images were collected under a laser scanning confocal microscope (Leica Microsystems GmbH, Wetzlar, Germany).

Short hairpin (sh) RNA transfection. shRNA plasmid vectors were purchased from Shanghai GenePharma Co., Ltd. (Shanghai, China), including pGPU6/GFP/Neo-shplexin-A1 (targeting plexin-A1) and pGPU6/GFP/Neo-shNC (not targeting any gene, which served as control). The constructs were transfected into MGC803 cells with jetPRIME ${ }^{\circledR}$ (Polyplus-transfection ${ }^{\circledR}$ SA, Illkirch, France). The procedure of transfection was performed according to the manufacturer's protocol. Next, stable cell clones were selected by treatment with 400-1,000 $\mu \mathrm{M}$ G418 (Beijing Solarbio Science \& Technology Co., Ltd.) for 1 month. Antibiotic-resistant cell clones were verified by the expression of green fluorescent protein.

Statistical analysis. All experiments were repeated $\geq 3$ times, unless otherwise indicated. Data are presented as the mean \pm standard deviation. Statistical analysis involved the use of one-way analysis of variance and Student's $t$-test. Statistical analysis was performed using SPSS version 19.0 (IBM SPSS, Armonk, NY, USA). P<0.05 was considered to indicate a statistically significant difference.

\section{Results}

Sema6D and its receptor plexin-Al are highly expressed in gastric tumor tissues compared with normal gastric mucosa. In the present study, RT-qPCR was performed to analyze four cases of gastric carcinoma and the corresponding cancer-adjacent lesions in gastric cancer. The results demonstrated that gastric tumor tissues expressed high levels of Sema6D compared with normal gastric mucosa (Fig. 1A). The protein expression levels of Sema6D were evaluated by western blotting (Fig. 1B). Based on the results of our current and previous studies (13), we speculated that Sema6D may serve a role in the formation and development of gastric cancer. According to a previous study, plexin-A1, plexin-A2 and plexin-A4 are all downstream receptors for Sema6D (22). Plexin-As have been shown to form a functional complex with neuropilin-1 (NP-1) and/or NP-2 (23) for certain semaphorins. For example, plexin-As are co-expressed with both Sema3A and NPs in yolk sac endothelial cells during vasculogenesis (24). However, NPs neither bound to Sema6D nor influenced the binding of Sema6D to plexin-A1, and another study also indicated that NPs may function independently (25). Based on the fact that Sema6D functions through plexin-As but does not depend on NPs, the present study next detected the levels of plexin-As in GES-1 cells and in a panel of gastric cancer cell lines by RT-PCR (Fig. 2A). The results indicated that plexin-A1 is highly expressed in gastric cancer cells lines compared with GES-1 cells. However, no significant difference in the mRNA expression of plexin-A2 

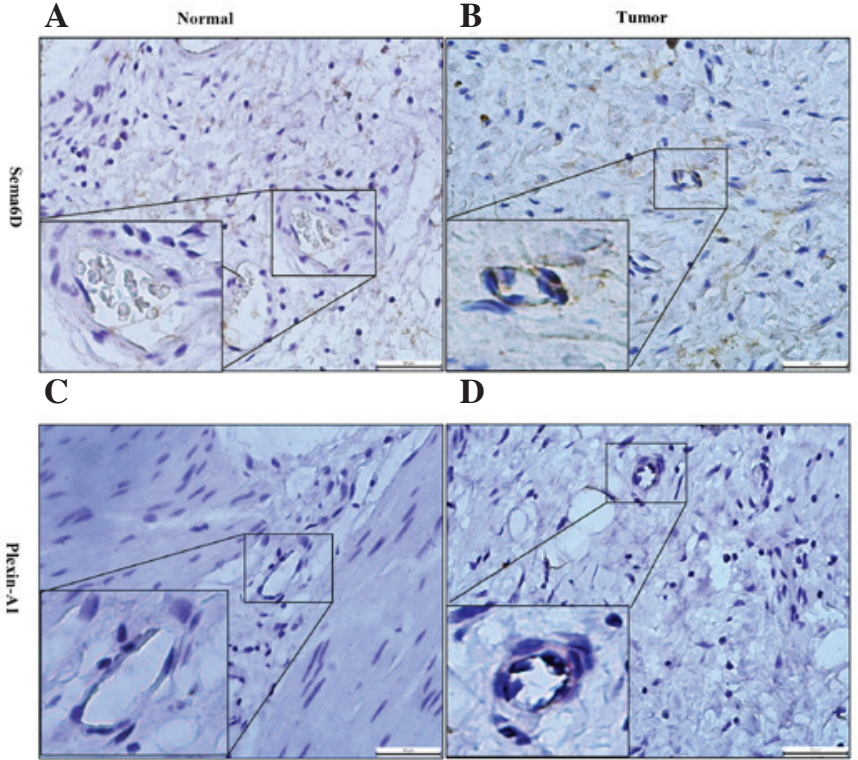

$\mathbf{E}$

$\mathbf{F}$

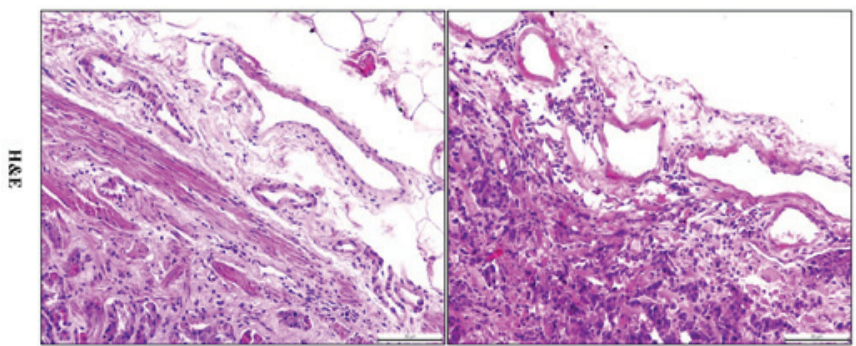

Figure 4. Sema6D and plexin-A1 were highly expressed in tumor vascular cells. (A and B) Immunohistochemical analysis revealed that, (A) compared with normal gastric tissue, (B) Sema6D (which appeared as brown particles) was highly expressed in the vascular endothelial cells within gastric cancer tissue. (C and D) Similar results were obtained for the expression of plexin-A1, which, (C) compared with normal gastric tissue, (D) was higher in gastric cancer vascular endothelial cells, and also appeared as brown particles. (E and F) H\&E staining revealed the morphological changes and depth of tumor invasion in (E) peri-cancerous and (F) cancerous tissues. Bar $=50 \mu \mathrm{m}$. Sema, semaphorin; H\&E, hematoxylin and eosin.

or plexin-A4 was identified between gastric cancer cells and normal gastric mucosa. It was also observed that plexin-A1 expression was remarkably higher than that of plexin-A2 and plexin-A4 in the gastric cancer cell lines (Fig. 2B). Based on previous studies $(13,19)$ and the current results, we speculate that plexin-A1 may be the putative receptor for Sema6D. Given the active roles of Sema6D in regulating tumor development observed in the present study, the mRNA and protein levels of plexin-A1 were next detected by qPCR and western blotting, respectively, using four cases of gastric cancer tissues and corresponding cancer-adjacent tissues. The results revealed that plexin-A1 was highly expressed in gastric tumor tissues at the mRNA level (Fig. 3A). Similar results were obtained for the plexin-A1 protein expression by western blotting (Fig. 3B). Therefore, we hypothesized that Sema6D functions in tumorigenesis using plexin-A1 as a receptor.

Sema6D and its receptor plexin-Al are highly expressed in vascular epithelial cells within gastric cancer. To further address the role of Sema6D and its receptor plexin-A1 in tumorigenesis, immunohistochemical methods were used to
A
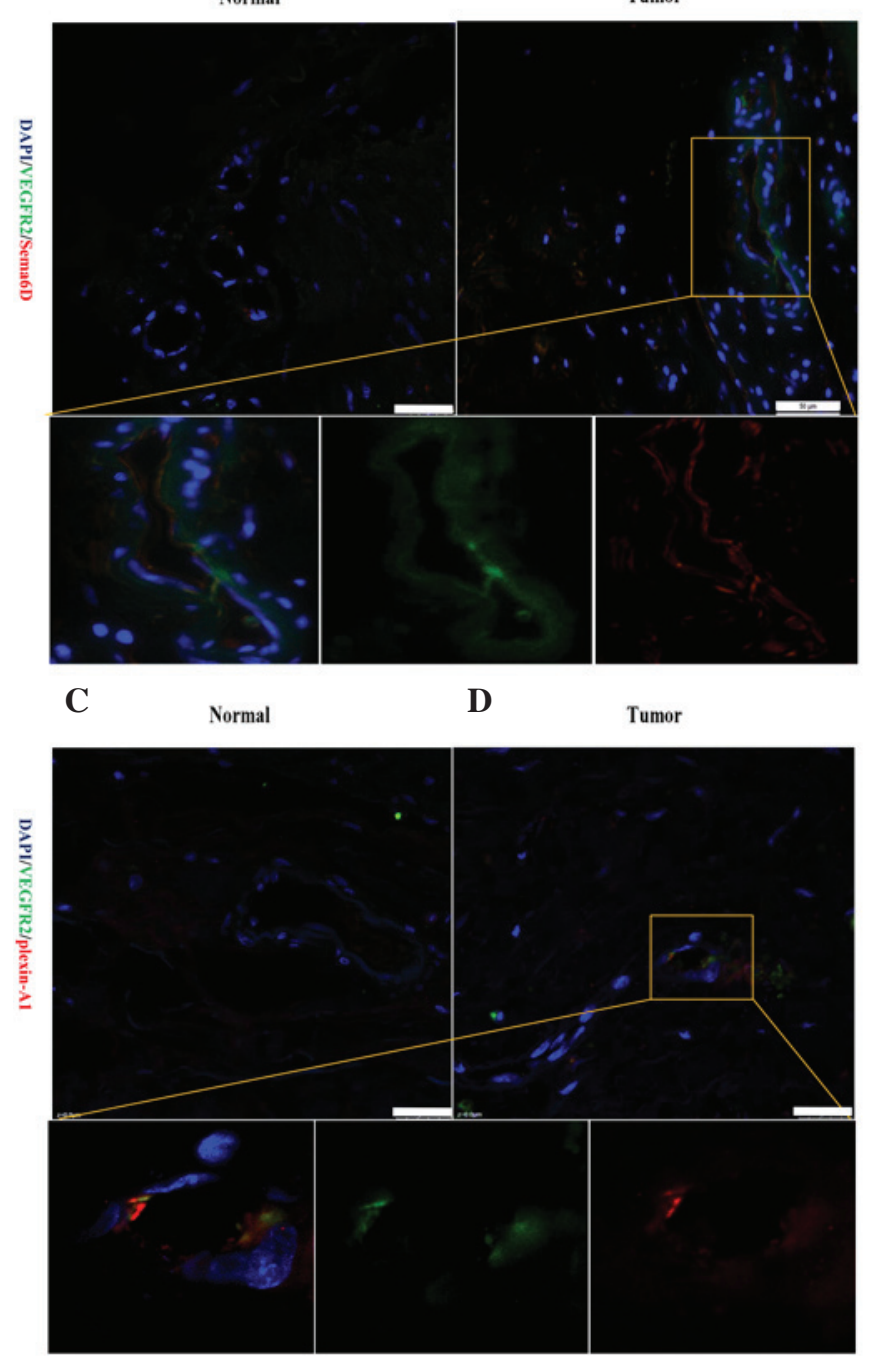

Figure 5. Sema6D and plexin-A1 were expressed and positively correlated with VEGFR2 in vascular endothelial cells. (A and B) Representative fluorescence images showing the double-labelling of Sema6D and VEGFR2 in (A) peri-cancerous and (B) cancerous vessels. In the cancerous tissues, Sema6D was upregulated and present in the vascular epithelial cells. Furthermore, Sema6D expression was strongly correlated with VEGFR2 expression in vascular epithelial cells. (C and D) Compared with (C) normal vascular cells within gastric cancer, the double-labelling of cells for plexin-A1 and VEGFR2 could also be detected in (D) vascular epithelial cells. Bar $=25 \mu \mathrm{m}$. Sema, semaphorin; VEGFR, vascular endothelial growth factor receptor; DAPI, 4',6-diamidino-2-phenylindole.

detect their expression and localization. Notably, Sema6D and plexin-A1 were mainly located at the membrane and cytoplasm of gastric carcinoma vascular endothelial cells, which appeared as brown particles (Fig. 4). Therefore, we speculated that they may serve a critical role in tumor angiogenesis. Angiogenesis involves the formation of new blood vessels from primitive vasculature, which is critical to numerous physiological processes, and vascular malformation may lead to several major diseases, particularly tumor progression (26). Thus, the present study next attempted to determine the mechanism of angiogenesis promotion mediated by Sema6D and plexin-A1 in vessel endothelial cells.

Sema6D and plexin-A1 promote tumor angiogenesis via VEGFR2 signaling. Various GFRs are implicated in 
A
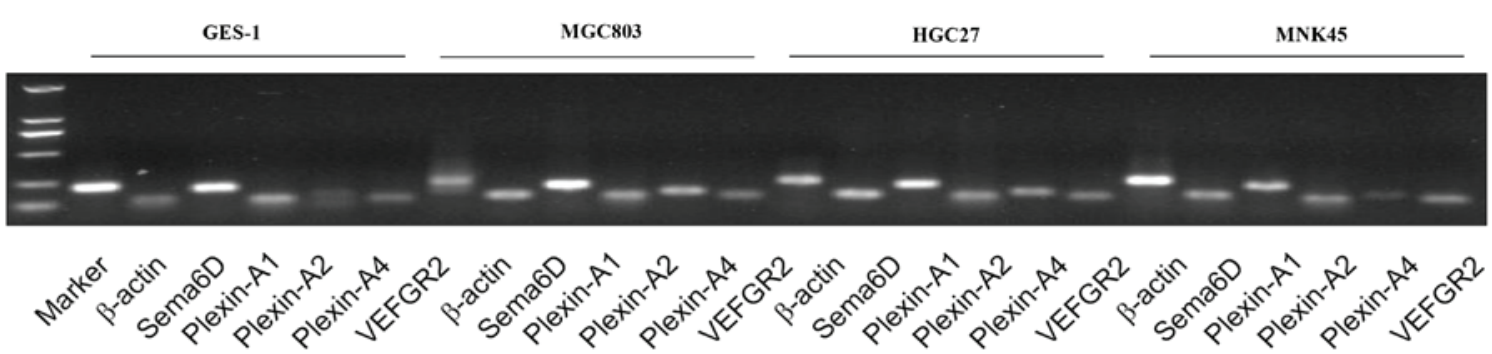

B

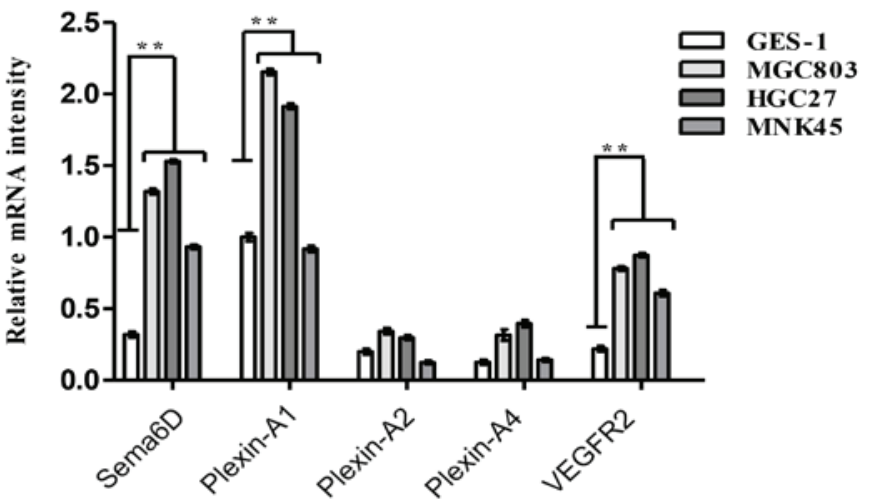

C

D

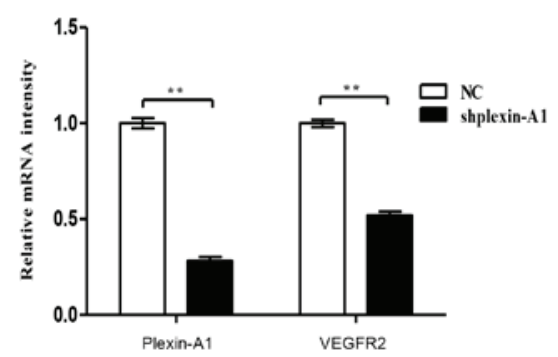

$\mathbf{E}$

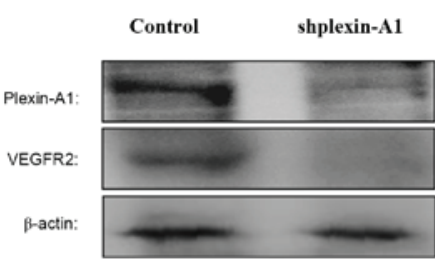

F

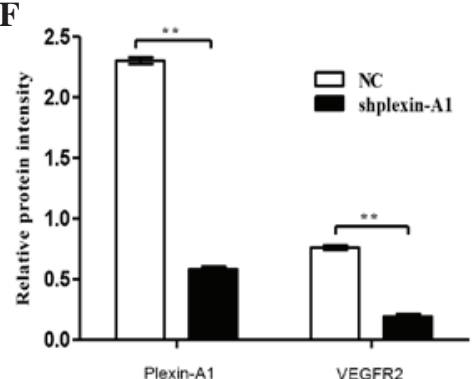

Figure 6. Sema6D and plexin-A1 promote angiogenesis through VEGFR2 signaling. (A) RT-PCR analysis detected the mRNA expression levels of Sema6D, plexin-As and VEGFR2 in three gastric cancer cell lines and GES-1 cells. (B) The histogram indicates the relative mRNA expression levels, which were normalized to the levels of $\beta$-actin. (C) Western blot analysis demonstrated that the protein expression levels of VEGFR2 were remarkably enhanced in MGC803 gastric cancer cells compared with GES-1 cells. (D) RT-quantitative PCR analysis of the mRNA expression levels of plexin-A1 and VEGFR2 upon using shplexin-A1 transfection to knock down plexin-A1. The mRNA expression level of VEGFR2 was downregulated. (E) Western blot analysis detected the protein levels of plexin-A1 and VEGFR2, and the protein expression of VEGFR2 was decreased with targeted disruption of plexin-A1. (F) The relative protein expression levels of plexin-A1 and VEGFR2 is shown. Data are represented as the mean \pm standard deviation ( $\mathrm{n}=3$ for each group, ${ }^{* *} \mathrm{P}<0.01$ ). Sema, semaphorin; VEGFR, vascular endothelial growth factor receptor; mRNA, messenger RNA; NC, negative control; sh, short hairpin; RT-PCR, reverse transcription-polymerase chain reaction.

angiogenesis, particularly the VEGFR family of receptor tyrosine kinases (27). VEGFR1 and VEGFR2 are closely related receptor tyrosine kinases, and VEGFR2 serves a broader role than VEGFR1 in angiogenesis (28). Sema6D was reported to participate in cardiac morphogenesis by exerting distinct biological activities through its receptor, plexin-A1, which formed receptor complexes with VEGFR2 in adjacent regions to the cardiac tube (15). Thus, the current study next detected the association of Sema6D and plexin-A1 with VEGFR2. An immunofluorescence method was used to detect the location of Sema6D and plexin-A1 in regards to that of VEGFR2. Notably, Sema6D and VEGFR2 were observed to be highly expressed in the membrane and cytoplasm of the same vascular endothelial cells in cancerous tissue. Representative fluorescence images revealed that Sema6D was expressed at a high level and was strongly correlated with the distribution patterns of VEGFR2 in tumor tissue (Fig. 5A and B). Similarly, double-labelling of cells for plexin-A1 and VEGFR2 could also be detected in the section of cancer tissues (Fig. 5C and D). This confirms that Sema6D/plexin-A1 may be closely associated with tumor angiogenesis via VEGFR2.

Sema6D, plexin-As and VEGFR2 were further systematically detected in three gastric cancer cell lines by RT-PCR (Fig. 6A), and significant differences in the mRNA expression of Sema6D, plexin-A1 and VEGFR2 were identified in gastric cancer cells compared with normal gastric mucosa (Fig. 6B). The protein expression levels of VEGFR2 in MGC803 and GES-1 cells were detected by western blotting, which revealed that VEGFR2 exhibited a high expression in MGC803 cells (Fig. 6C). To further address the interaction between plexin-A1 and VEGFR2, plexin-A1 was knocked down using shplexin-A1 in MGC803 cells. The mRNA (Fig. 6D) and protein (Fig. 6E and F) expression levels of VEGFR2 were downregulated with the targeted disruption of plexin-A1. This finding verified our speculation that plexin-A1 serves its role via VEGFR2 in tumor angiogenesis. 


\section{Discussion}

Gastric cancer is the fifth most common cancer and the third leading cause of cancer mortality worldwide, with nearly 950,000 new cases and 723,000 mortalities estimated in 2012 (29). Angiogenesis involves the formation of new blood vessels, which serves a pivotal role in tumor growth and metastasis (30). Anti-angiogenic therapies targeting VEGF and its receptors have been developed in recent years (31). However, in gastric cancer, the therapeutic role of anti-angiogenic agents remains to be determined, particularly for patients with advanced disease, where treatment options are limited.

Despite the fact that Sema6D and plexin-A1 serve a crucial role in the formation of the nervous system, increasing evidence suggests their participation in cardiogenesis (15). Ferrara and Kerbel indicated that the action of VEGF is mainly mediated through VEGFR2, which is present in tumor endothelial cells (32). Therefore, the present study aimed to investigate the interaction of Sema6D/plexin-A1 and VEGFR2.

In conclusion, the current study has provided evidence that Sema6D and plexin-A1 may regulate angiogenesis in vitro, and raises the possibility that they may serve a role in tumor-induced angiogenesis by VEGFR2. Further studies will be necessary to elucidate whether there is a secreted factor responsible for the tumor-promotion effect of Sema6D and plexin-A1 in gastric carcinoma, such as VEGFR2, and to determine whether other members of the semaphorin family and their receptors can function as tumor stimulators or suppressors in vivo.

Understanding the function of Sema6D and plexin-A1 in the regulation of malignant transformation may aid to unravel the molecular mechanisms involved in gastric cancer, which may open novel therapeutic avenues to interfere with this process. Our studies indicated a direct connection between Sema6D, plexin-A1 and gastric cancer angiogenesis, and may open novel therapeutic avenues to interfere with this process. Therefore, future clinical trials may be undertaken with the blockage of the Sema6D/plexin-A1 pathway for the treatment of gastric cancer regarding angiogenesis, which serves an important pathogenic role.

\section{Acknowledgements}

The present study was supported by the Key Subjects in Universities and Colleges of Hebei Province of China (Pathology and Pathophysiology; Shijiazhuang, China) and the Science and Technology Support Program of Hebei Province of China (Shijiazhuang, China; grant no. 13277779D).

\section{References}

1. Kolodkin AL, Matthes DJ and Goodman CS: The semaphorin genes encode a family of transmembrane and secreted growth cone guidance molecules. Cell 75: 1389-1399, 1993.

2. Takahashi K, Ishida M, Hirokawa K and Takahashi $\mathrm{H}$ : Expression of the semaphorins Sema 3D and Sema 3F in the developing parathyroid and thymus. Dev Dyn 237: 1699-1708, 2008.

3. Behar O, Golden JA, Mashimo H, Schoen FJ and Fishman MC: Semaphorin III is needed for normal patterning and growth of nerves, bones and heart. Nature 383: 525-528, 1996.

4. Gitler AD, Lu MM and Epstein JA: PlexinD1 and semaphorin signaling are required in endothelial cells for cardiovascular development. Dev Cell 7: 107-116, 2004.
5. Gu C, Rodriguez ER, Reimert DV, Shu T, Fritzsch B, Richards LJ, Kolodkin AL and Ginty DD: Neuropilin-1 conveys semaphorin and VEGF signaling during neural and cardiovascular development. Dev Cell 5: 45-57, 2003.

6. Suzuki K, Kumanogoh A and Kikutani H: Semaphorins and their receptors in immune cell interactions. Nat Immunol 9: 17-23, 2008.

7. Tse C, Xiang RH, Bracht T and Naylor SL: Human Semaphorin 3B (SEMA3B) located at chromosome 3p21.3 suppresses tumor formation in an adenocarcinoma cell line. Cancer Res 62: 542-546, 2002

8. Tomizawa Y, Sekido Y, Kondo M, Gao B, Yokota J, Roche J, Drabkin H, Lerman MI, Gazdar AF and Minna JD: Inhibition of lung cancer cell growth and induction of apoptosis after reexpression of 3p21.3 candidate tumor suppressor gene SEMA3B. Proc Natl Acad Sci USA 98: 13954-13959, 2001.

9. Miao HQ, Soker S, Feiner L, Alonso JL, Raper JA and Klagsbrun M: Neuropilin-1 mediates collapsin-1/semaphorin III inhibition of endothelial cell motility: Functional competition of collapsin-1 and vascular endothelial growth factor-165. J Cell Biol 146: 233-342, 1999.

10. Kigel B, Varshavsky A, Kessler O and Neufeld G: Successful inhibition of tumor development by specific class-3 semaphorins is associated with expression of appropriate semaphorin receptors by tumor cells. PLoS One 3: e3287, 2008

11. Banu N, Teichman J, Dunlap-Brown M, Villegas G and Tufro A: Semaphorin 3C regulates endothelial cell function by increasing integrin activity. FASEB J 20: 2150-2152, 2006.

12. Basile JR, Castilho RM, Williams VP and Gutkind JS: Semaphorin 4D provides a link between axon guidance processes and tumor-induced angiogenesis. Proc Natl Acad Sci USA 103: 9017-9022, 2006.

13. Zhao XY, Chen L, Xu Q and Li YH: Expression of semaphorin 6D in gastric carcinoma and its significance. World Gastroenterol 12: 7388-7390, 2006.

14. O'Connor BP, Eun SY, Ye Z, Zozulya AL, Lich JD, Moore CB, Iocca HA, Roney KE, Holl EK, Wu QP, et al: Semaphorin $6 \mathrm{D}$ regulates the late phase of CD4+ T cell primary immune responses. Proc Natl Acad Sci USA 105: 13015-13020, 2008.

15. Toyofuku $\mathrm{T}$, Zhang $\mathrm{H}$, Kumanogoh A, Takegahara $\mathrm{N}$ Suto F, Kamei J, Aoki K, Yabuki M, Hori M, Fujisawa H and Kikutani H: Dual roles of Sema6D in cardiac morphogenesis through region-specific association of its receptor, Plexin-A1, with off-track and vascular endothelial growth factor receptor type 2. Genes Dev 18: 435-447, 2004.

16. Moriarity BS, Otto GM and Rahrmann EP, Rathe SK, Wolf NK, Weg MT, Manlove LA, LaRue RS, Temiz NA, Molyneux SD et al: A Sleeping Beauty forward genetic screen identifies new genes and pathways driving osteosarcoma development and metastasis. Nat Genet 47: 615-624, 2015.

17. Toyofuku T, Zhang H, Kumanogoh A, Takegahara N, Yabuki M, Harada K, Hori M and Kikutani H: Guidance of myocardial patterning in cardiac development by Sema6D reverse signalling. Nat Cell Biol 6: 1204-1211, 2004

18. Kigel B, Rabinowicz N, Varshavsky A, Kessler O and Neufeld G: Plexin-A4 promotes tumor progression and tumor angiogenesis by enhancement of VEGF and bFGF signaling. Blood 118: 4285-4296, 2011

19. Zhao XY, Chen L, Li YH and Xu Q: PlexinA1 expression in gastric carcinoma and its relationship with tumor angiogenesis and proliferation. World J Gastroenterol 13: 6558-6561, 2007.

20. Catalano A, Lazzarini R, Di Nuzzo S, Orciari S and Procopio A: The plexin-A1 receptor activates vascular endothelial growth factor-receptor 2 and nuclear factor-kappaB to mediate survival and anchorage-independent growth of malignant mesothelioma cells. Cancer Res 69: 1485-1493, 2009.

21. Livak KJ and Schmittgen TD: Analysis of relative gene expression data using real-time quantitative PCR and the 2(-Delta Delta C(T)) Method. Methods 25: 402-408, 2001.

22. Suzuki K, Kumanogoh A and Kikutani H: Semaphorins and their receptors in immune cell interactions. Nat Immunol 9: 17-23, 2008.

23. Fujii T, Nakao F, Shibata Y, Shioi G, Kodama E, Fujisawa H and Takagi S: Caenorhabditis elegans PlexinA, PLX-1, interacts with transmembrane semaphorins and regulates epidermal morphogenesis. Development 129: 2053-2063, 2002.

24. Herzog Y, Kalcheim C, Kahane N, Reshef R and Neufeld G: Differential expression of neuropilin-1 and neuropilin-2 in arteries and veins. Mech Dev 109: 115-119, 2001 
25. Ellis LM: The role of neuropilins in cancer. Mol Cancer Ther 5 : 1099-1107, 2006.

26. Peplow PV: Influence of growth factors and cytokines on angiogenic function of endothelial progenitor cells: A review of in vitro human studies. Growth Factors 32: 83-116, 2014.

27. Meunier-Carpentier S, Dales JP, Djemli A, Garcia S, Bonnier P, Andrac-Meyer L, Lavaut MN, Allasia C and Charpin C: Comparison of the prognosis indication of VEGFR-1 and VEGFR-2 and Tie2 receptor expression in breast carcinoma. Int J Oncol 26: 977-984, 2005.

28. Rahimi N: VEGFR-1 and VEGFR-2: Two non-identical twins with a unique physiognomy. Front Biosci 11: 818-829, 2006.

29. Ferlay J, Soerjomataram I, Dikshit R, Eser S, Mathers C, Rebelo M, Parkin DM, Forman D and Bray F: Cancer incidence and mortality worldwide: Sources, methods and major patterns in GLOBOCAN 2012. Int J Cancer 136: E359-E386, 2015.
30. Zhang ZL, Liu ZS and Sun Q: Effects of thalidomide on angiogenesis and tumor growth and metastasis of human hepatocellular carcinoma in nude mice. World J Gastroenterol 11: 216-220, 2005

31. Bertolini F, Marighetti P, Martin-Padura I, Mancuso P, Hu-Lowe DD, Shaked Y and D'Onofrio A: Anti-VEGF and beyond: Shaping a new generation of anti-angiogenic therapies for cancer. Drug Discov Today 16: 1052-1060, 2011.

32. Ferrara N and Kerbel RS: Angiogenesis as a therapeutic target. Nature 438: 967-974, 2005. 